\title{
Verhandlungen der Abteilung für Dermatologie und Syphilis der 74. Versammlung Deutscher Naturforscher und Ärzte in Karlsbad
}

vom 22.-27. September 1902.

Erstattet von Dr. Ignaz Böhm, Karlsbad.

I. Sitzung am 22. September, nachmittags 3 Uhr.

Die Sektion für Dermatologie und Syphilis, die unter den vielversprechenden Auspizien der Klinik des Prof. F. J. Pick stand, wurde von dem Karlsbader Finführenden Böhm mit folgender Ansprache begrüßt und eröffnet:

Es ist mir die besondere Ehre zu teil geworden, Sie, meine geehrten Ferren, die aus der Ferne zu uns gekommen sind, aufs herzlichste zu begrüßen und willkommen zu heißen. Willkommen in Karlsbad, dem durch seine wunderbaren Heilquellen weltbekannten Kurorte, willkommen zu ernstem Schaffen und Denken, aber auch zu fröhlichem, kollegialen Verkehre. Mehr denn vier Dezennien sind verstrichen seit der ersten Tagung der Versammlung Deutscher Naturforscher und Ärzte in unserer Stadt, und nach einem so großen Intervalle haben wir wieder das Glück, in Verbindung mit der diesjährigen Versammlung, die Sektion für Dermatologie hier tagen zu sehen. Es liegt mir ferne, die Entdeckungen und Errungenschaften dermatologischer Forschung in diesem Zeitraume zu würdigen, ich will bloß mit Rücksicht auf unseren Kurort feststellen, daß die Erkenntnis des Zusammenbanges zwischen internen und dermatologischen Erkrankungen in dieser Zeitperiode wesentlich gefördert worden ist. Die häufigsten zum Kurgebrauche in Karlsbad gelangenden Erkrankungen sind Stoffwechselstörungen, Autointoxikationen und nicht sehr selten sind durch dieselben Veränderungen auf der Haut provoziert. Dadurch, daß die hiesigen Heilquellen diese internen Erkrankungen einer Heilung oder Besserung zuführen, werden auch die sekundären und symptomatischen Hautaffektionen im günstigsten Sinne beeinflußt. Sie sind deshalb, meine geehrten Herren, in einem Ihnen in seiner Wirkung auf die Haut bereits wohlbekannten Kurorte zu ernster, wissenschaftlicher Arbeit erschienen. Der gute Klang, den der Name Karlsbad in medizini- 
schen Kreisen hat, hat auch auf unsere Sektion in vorteilhafter Weise eingewirkt, denn die Zahl der angemeldeten Vorträge, die starke Beteiligung legen hiefür das beste Zeugnis ab. Möge die ernste Arbeit, die Sie hier leisten werden, mögen die Resultate Ihrer Forschungen, die Sie hier zum ersten Male kundtun, zu Nutz und Frommen der leidenden Menschheit werden, mögen die Erfolge unserer Sektion von derartiger Bedeutung sein, daß mit Stolz Karlsbad genannt werden kann als der Ort, an dem sie zum ersten Male mitgeteilt wurden.

Hierauf übernimmt den Vorsitz Herr Prof. Ehrmann, Wien.

Die Reihe der wissenschaftlichen Arbeiten eröffneten

Ledermann und Kloppstock (Berlin) mit dem Vortrage: Die bakterizide Wirkung verschiedener Hefepräparate. In neuerer Zeit hat Lassar Versuche über die Heilwirkung der Hefe bei Hautkrankheiten mit günstigen Erfolgen angestellt, ebenso Brocq, der den internen Gebrauch derselben bei Antrax, Furunculose empfiehlt. Die Vortragenden hielten es daher für lohnend, die Frage der bakteriziden Hefewirkung auch vom dermatologischen Standpunkte aus zu beantworten und hofften, daß der Tierversuch eine Antwort auf die schwebenden Fragen geben würde. Alle angestellten Versuche sind jedoch deswegen gescheitert, weil man den Tieren nicht analoge Hautkrankheiten beibringen kann. Es ließ sich feststellev, daß die Hefe auch bei längerer interner Darreichung nicht den geringsten Schaden verursachte. Wurde Kaninchen, welchen vorher durch Staphylokokkeninfektion subkntane Abszesse beigebracht waren, nachträglich an anderen Stellen Hefe mit Gährflüssigkeit subkutan injiziert, so war der Erfolg der, daß nun auch durch die gährende Hefe neue Abszesse erzeugt wurden. Sie verglichen bei dem ersten Versuche die Einwirkung der gährenden Dauerhefe, des Furunculins und einer aus Bierhefe schon längere Zeit fortgezüchteten Reinkultur auf Typhusbazillen und fanden, daß nach 48 Stunden die in der Dauerhefe eingeimpften Typhusbazillen abgetötet waren, während Furunculin und die Hefekultur das Bakterienwachstum unbeeinflußt gelassen haben. Kontrollversuch ohne Hefezusatz. Ein zweiter, analoger Versuch wurde dadurch vervollständigt, daß außer den genannten Hefepräparaten noch die sogenannte Preßhefe der Bäcker mit herangezogen wurde und ebenso wie die Dauerhefe in diesem Versuche schon nach 28 Stunden das Wachstum der Typhusbazillen veruichtete. Alle verwendeten Hefearten waren gährungsfähig. In einem zweiten Versuche wurde die Einwirkung der genannten vier Hefepräparate auf Staphylokokken geprüft und dabei ergab sich, daß bei Einimpfung in Dauerhefe die Staphylokokken nach 54 Stunden abgetötet waren, während bei Einwirkung von Preßhefe schon nach 6 Stunden eine deutliche Wachstumsverminderung, nach 24 Stunden eine vollständige Vernichtung der Keime beobachtet warde. Bei einem Kontrollversuche tötete die gährende Dauerhefe schon nach 48 Stunden sämtliche Staphylokokkenkeime ab, während Furunculin und die Bierhefekultur keinen Einfuß auf das Wachstum ausübte. Die Preßhefe beeinflußte das Wachstum der Staphylokokken derart, daB von anfangs unzähligen Keimen nach 54 Stunden noch 40 Keime auf der Platte übrig blieben, die nur aus Hefezellen bestanden, so daß auch hier eine Abtötung der Staphylokokken stattfand. Bacterium coli wurde erst nach längerer Zeit (72-76 Stunden) abgetötet, ist also widerstandsfähiger als die anderen Bakterien gegenüber Dauerhefe. Furunculin und Hefekultur ließen in zwei Versuchen das Bakterienwachstum unverändert. Was nun das Resultat der Untersuchungen anlangt, so läßt sich eine entwicklungshemmende bakterizide Wirkung der gährenden Dauerhefe, Preßhefe und Levurinose auf Typhusbazillen, Staphylokokken und Bacterium coli im 
Glase nachweisen. Die zur Abtötung erforderliche Zeit schwankte zwischen 24 und 76 Stunden. Die bakterizide Wirkung fehlt vollkommen dem Furunculin und geht auch der aus Bierhefe gezüchteten Hefereinkultur ab. Wenn es nun wahr ist, daß per os verabreichte Hefe eine Heilwirkung auf Furunkel, Akne etc. hat, so kann dies nur so erklärt werden, daß bei der im Darme vor sich gehenden Hefegährung lösliche Produkte entstehen, welche resorbiert ins Blut gelangen, von dort den Krankheitsherden zugeführt werden, um dort das Bakterienwachstum zu hemmen oder zu vernichten. Bei der Hefekur ist der Nahrung besondere Aufmerksamkeit zuzuwenden, da bei Verabreichung von Reis, Sago, Gries die Heilwirkung größer ist als bei Fleischkost.

Herz (von der Prager Hautklinik) teilt die Ergebnisse seiner Versuche über Agglutination der Tuberkelbazillen bei Hauttuberkulose mit.

Die Versuche wurden mit der von Koch angegebenen Testflüssig. keit (einer Emulsion von getrockneten und zu feinstem Staub zerriebenen Tuberkelkulturen in einer $0.5 \%$ Karbolsäure und $0.85 \% \mathrm{NaCl}$ Lösung) angestellt. Fis wurde das Serum von 31 Fällen von mehr weniger hochgradiger Haut- und Schleimhanttuberkulose sowie eines Falles vou Lichen scrophulosum zu wiederholten Untersuchungen benützt. Die Resultate erweisen die Unverwendbarkeit der Serumreaktion als diagncstisches Hilfsmittel bei der Hanttuberkulose, dagegen scheint der Ausfall der Reaktion einen Mabstah für die natüriche oder künstliche Immunisierung des Körpers gegen T'uberkulose zu bilden.

Winternitz (Prag): Eine Trichomykosis des behaarten Kopfes.

Nach Besprechung einiger bloß das Haar betreffenden infektiöser Erkrankungen (Piedra, Behrend, Unua), (Palmellina, Pick, Colombini), (Trichorrhexis nodosa Hodara, Spiegler) führt W. an der Hand von Abbildungen und mikroskopischen Präparaten das Bild einer wohl sehr vereinzelten Haarerkrankung vor. Sitz derselben war der behaarte Kopf, ergriffen war eine größere Anzahl von Haaren an der Schläfe und der hinteren Haargrenze. Die betreffenden Haare zeigten Verdickungen, respektive bis $1 / 2 \mathrm{~cm}$ lange Finscheidungen, die aus Pilzmassen und zwar aus strahlig verlaufenden Fäden bestanden, welche letzteren sich in Bazillen auflösen ließen. Diese wurden reingezüchtet und zeigten Besonderheiten gegenüber den bisher bei infektiösen Haarerkrankungen (Piedra, Palmellina, Trichorrhexis) gefundenen und gezüchteten.

Diskussion: Pick (Prag) betont das Vorkommen der Trichomycosis palmelliar an denselben Stellen, wie die von W. angeführten und weist deshalh auf die Notwendigkeit und anf die Möglichkeit einer Differenzierung dieser Mykose von der Mycosis palmellina hin.

Winternitz (Schlußwort): Bei dem Umstande, als die Auflagerungen am Haare, wie die mikroskopischen Präparate zeigen, blob aus Bazillen bestehen und bei der /ächtung nur letztere in Reinkultur aufgehen, ist die Ätiologie wobl geklärt. Trotzdem ist eine Überimpfung auf das Haar noch nötig und hat $W$. eine solche auf dem Wollhaar seines eigenen Armes, aber erst in der letzten Woche gemacht. Fr wird später über die Impfungen berichten.

Saalfeld (Berlin): Diabetes und Hautkrankheiten.

Hautaffektionen, die ansschlieblich dem Diabetes zukommen, sind das Xanthoma diabeticum, der Diabete broncé, die Gangraena diabetica, die Gangraena diabetica bullosa serpiginosa und die Dermatitis diabetica papillomatosa. Die bei Diabetikern vorkommenden Fälle vou Mal per- 
forant du pied lassen sich hier anreihen. Im Gegensatz zu den eben geschilderten Leiden geht $\mathrm{S}$. nun auf diejenigen Hauterkrankungen ein, die als zufällige Begleiterscheinungen mit dem Diabetes koinzidieren. E $\mathbf{r}$ wies zunächst auf die Neigung zur Nekrosenbildung, respektive die schwere Heilbarkeit bei den durch Mikroorganismen hervorgerufenen entzündlichen Prozessen der Haut hin, wie Furunkel, Karbunkel, Paronychie, einen von $\mathrm{ihm}$ beobachteten Fall von Acne necrotisans. Die Abhängigkeit ist weiters daraus ersichtlich, daß die Erkrankungen mit dem Heruntergehen des Zuckergehaltes gleichfalls sich bessern, respektive zur Heilung gelangen. Analoges beobachtete $S$. bei drei Fällen von Acne rosacea, einem Falle von Ulcus molle, ferner von Ulcus cruris traumaticum, während er den Herpes zoster bei Diabetikern mit besonders großen Schmerzen und einmal mit Gangränbildung verlaufen sah. Pruritus universalis und localis ist bei Diabetikern ungemein häufig. Eczema et Pruritus scroti und vulvae, Eczema intertrigo, Balanitis mit Neigung zur Phimosis sind allgemein bekannt. Von den sonst noch mit Diabetes mehr oder weniger in direktem Zusammenhange stehenden Dermatosen hob $S$ die Anidrosis und Asteatosis, die viel seltenere Hyperidrosis, die Urticaria und das Erythem hervor, während er bei seinen Psoriasisfällen niemals Glykosurie beobachten konnte. Hauterkrankungen als prämonitorisches Zeichen für den Diabetes sah S. in zwei Fällen. Im Anhange äußerte sich Vortragender über den Zusammenhang des Diabetes mit der Lues. Beide Krankheiten können einfach koinzidieren oder die Glykosurie kann eine Folge der Lues sein. Letzteres ist der Fall, wenn beide Affektionen an einem Patienten auftreten, der früher nicht an Diabetes gelitten hat, wenn die Glykosurie unter der antisyphilitischen Behandlung zurückgeht ohne antidiabetische Diät, und wenn bei einem Luesrezidiv sich wieder Zucker zeigt. Andererseits kann gerade umgekehrt bei Einleitung einer Hg-Kur sich Zucker einstellen, der dann nach Unterbrechung derselben aufhört oder auch nicht, wie $S$. in einem Falle beobachten konnte. Ferner sah er in zwei Fällen einen günstigen Einfluß der $\mathrm{Hg}$ einreibung auf den Diabetes insipidus. Für die erfolgreiche Behandlung der Hauterkrankungen bei Diabetes ist die allgemeine Therapie eine conditio sine qua non.

Diskussiou: Demetriade (Jassy) erwähnt einen Fall mit einem Geschwüre am Skrotum, bei welchem er einen Diabetes als Ursache fest. stellen konnte. Err betont das häufige Zusammentreffen von Lues und Diabetes.

Böhm (Karlsbad) berichtet von einem 42jährigen Kurgaste mit Diabetes mellitus $(4-5 \%)$ und Psoriasis prof. univ. Die Psoriasis war von so starker Ausbreitung, daß am ganzen Rumpfe kein Plätzchen gesunder Haut zu finden war. Mit der Abnahme des Zuckers besserte sich auch der schwere Zustand der Psoriasis. Ob der Diabetes die Ursache der Psoriasis ist, kann nicht entschieden werden, aber so viel steht fest, daß der Diabetes zeitlich früher aufgetreten ist als die Psoriasis.

Justus (Budapest) berichtet von einer 40jährigen Patientin, die ein syphilitisches Ulcus des linken Unterschenkels, eine Atrophie der Nervi optici und einen Diabetes insipidus hatte mit 10-12 Liter täglicher Harnmenge. Unter $\mathrm{Hg}$-Kur trat Heilung des Uleus und Abnahme des Harnes (2 Liter) ein. Atrophie blieb unverändert. Nach einigen Monaten Rezidiv des Diabetes insipidus. 
Siebel (Karlsbad) macht Mitteilung von einem Diabetesfalle, der durch Karlsbader Kur nicht znckerfrei wurde, durch eine Inunktionskur aber in drei Wochen den Zuckex verlor.

Pick (Prag) fügt einige Bemerkungen zu den Angaben des Vortragenden bezüglich der Koinzidenz von Diabetes und Psoriasis, weist auf die von seinem Sohne Walther Pick aus der Klinik Neisser mitgeteilten statistischen und experimentellen Angaben bezüglich der größeren Neigung der Psoriatischen zur alimentären Glykosurie hin, mit welchen die Resultate der zahlreichen, analogen Beobachtongen an seiner Klinik übereinstimmen.

Ledermann (Berlin) bericbtet von einem 6jährigen Kinde mit extragenitaler Luesinfektion und Diabetes insipidus; Jod, $\mathrm{Hg}$ ohne EinfluB, Exitus. Er weist dann auf die Schwierigkeiten hin, die entstehen, wenn Nephritis bei Lues und Diabetes während der Schmierkur eintritt. Zum Schlusse bespricht er die klinische Veränderung, die die Psoriasis der Genitale hei Frauen erleidet, indem die Haut stark glänzend, glatt wie bei Xeroderma erscheint und weist darauf hin, daß es sich bei Zusammenstellung von $\mathrm{Naglschmidt} \mathrm{nur} \mathrm{um} \mathrm{don} \mathrm{Nachweis} \mathrm{einer} \mathrm{alimentären}$ Glykosurie in einem gewissen Prozentsatze der Psoriasisfälle handelt.

Viktor Bandler (Prag) erwähnt seine Publikation vom Jahre 1898 aus der Klinik Pi ck, betreffend einen Fall von hereditärer Lues mit Diabetes insipidus. Harnmenge 18-20 Liter täglich. Eine eingeleitete Inunktionskur bewirkt Heilung.

Ehrmann (Wien) weist auf seine bereits publizierte Beobachtung hin, dab bei Diabetikern Erosionen am Genitale eine Beschaffenheit annehmen, welche sie der einer Sklerose ähnlich macht. Er bespricht dann noch die syphilitische Polyurie und den Diabetes bei Syphilis.

Saalfeld (Schlußwort) glaubt, daß Ledermann und Bandler ihn bezüglich der von ihm erwähnten Fälle von Diabetes insipidus mißverstanden baben. Bei dem einen Fall ging die Polydipsie und Polyurie zurück, als wegen einer frischen Lues eine IIg-Kur eingeleitet wurde; bei dem zweiten Falle ließen ebenfalls die Erscheinungen des Diabetes insipidus nach, als wegen Auftreten von Morpiones unquentum cinereum angewendet wurde, ohne dab Lues bestand.

Spiegler (Wien): Beiträge zur Kenntnis des Pigmentes.

Über den Ursprung des Pigmentes bestehen zwei entgegengesetzte Auffassungen: Die hämatogene Lehre einerseits und andererseits diejenige, der zufolge das Pigment ein Produkt der Zelltätigkeit ist ohne Mitwirken des Blutfarbstoffes. Die Frage ist nicht histologisch lösbar, sondern nur durch Darstellung und chemische Untersuchung großer Pigmentmengen. Der Vortrageude hat solche aus schwarzem Roßhaar dargestellt. Da sich aus diescon Pigment weder Hämopyrol noch Küs ters Hämatinsäuren darstellen lassen, ist ein Zusammenhang mit Blutfarbstoff ansgeschlossen. Aber auch aus Schimmelhar und weißer Wolle läBt sich ein helles P'igment gewinnen, das mit Ammoniak behandelt, schwarz wird und das in seiner Zusammensetzung dem schwarzen sehr ähnlich ist. Sowohl das belle als auch das schwarze Pigment geben ein identisches Oxydationsprodukt, welches schön krystallisiert und bei $69^{\circ} \mathrm{C}$. schmilat. Außerdem gibt das schwarze Pigment noch weiße Reduktionsprodukte, die sich durch Behandlung mit Eisessig und Zinkstaub, sowie durch elektrolytische 
Reduktion, schwer aber durch Reduktion mit alkalischen Reduktionsmitteln herstellen lassen.

Diskussion: Winternitz (Prag) fragt, ob der von S. dargestellte Farbstoff mil dem von Sieber aus Menschenbaaren und Haut dargestellten, respektive mit den von $\mathrm{Nenki}$ und Berdez aus einem Melanosarkom und aus Pferdehaar dargestellten identisch ist.

Spiegler verneint dies.

Winternitz: Auch Sieber hat bereits darauf hingewiesen, da $B$ zwischen dem Blut- und dem Haarfarbstoffe kein Zusammenhang besteht.

Löwenheim (Liegnitz) fragt, ob Untersuchungen über die Färbekraft gemacht wurden. Selbst wenn das Pigment nur in Schwefelsäure oder Kalilauge löslich ist, muß es sich doch ermöglichen lassen, zu beweisen, daß das demonstrierte Präparat ein Farbstoff ist.

Paul Richter (Berlin) : Über unschädliche Haarfärbemittel.

Im Jahre 1897 hat $R$. das Aureol, welches $\operatorname{Erdmann}$ erfunden, als unschädliches Haarfärbemittel empfohlen. Das bält er auch jetzt noch aufrecht, da das Aureol kein Paraphenylendiamin enthält, während der Fall von $\mathrm{Schütz}$, auf dessen Angriffe dieser Vortrag als Antwort dieneu soll, das paraphenylendiaminhalige "Juvenia" von Guesqu in in Paris betraf. Die seltenen Hautentzündungen nach Aureol sind lokal beschränkt und heilen leicht. Das darin enthaltene Metol, welches manchmal Hautentzündungen hervorruft, kann ohne weiteres fortgelassen werden.

Ehrmann (Wien): Über die Sklerodermie und ihre Beziehung zu den autotoxischen Erythemen.

E, teilt seine Arbeit in drei Teile, deren erster die tatsächliche Beobachtung an der Haut, deren zweiter die Koinzidenz mit anderweitigen Erkrankungen, namentlich mit Atonie des Darmes betrifft und deren dritter endlich die Schlußfolgerungen zieht, welche dazu führen, die Sklerodermie als eine autotoxische Erkrankung anzusehen. Im ersten Teile herichtet $\mathrm{H}$. über Fälle, bei welchen der Sklerodermie unmittelbar vorangehend und mit ihr gleichzeitig urticarielle Erytheme umschriebener Natur verliefen, dann allgemeine schwere Erytheme mit schwerer Allgemeinerkrankung, die direkt in Sklerodermie übergingen und von denen ein Fall letal endete. In diesem Falle war auch Skferodermie am weichen Gaumen und im Anfangsteile des Ösophagus, Nieren- und Lungenaffektion vorhanden. E. weist darauf hin, daB die circumscripten Sklerodermien fast alle aus Erythemflecken hervorgehen, beim Fortschreiten in der Peripherie immer von akuten Erythemen begleitet werden. Auss diesen Erythemen geht auch die Pigmentbildung hervor. Bezüglich der Ätiologie, die den dritten Teil der Besprechung bildet, findet E., daß bei einer großen Zahl seiner Fälle Störungen der Darmmotilität vorhanden sind. Er gibt wohl zu, daß die toxische Substanz auch anderweitig entstehen könne als im Darme, jedoch in seinen Fällen scheint zweifellos der Darm die Bereitungsstätte des Giftes zu sein. Die von Beer beobachtete Erscheinung, daß die umschriebene Sklerodermie an solchen Stellen sich zeigt, wo dauernder Druck oder Reibung von seiten der Bekleidung und Bewaffnung vorhanden ist, kann nicht so gedeutet werdea, daß jedes mechanisch erzengte Ekzem bei jedem Menschen in Sklerodermie übergehen kann, sondern nur so, daß bei solchen Menschen, welche der betreffenden toxischen Wirkung ausgesetzt sind, ähnlich wie bei Lues, Lichen planus etc. die Reizung eine frische Lokalisation an der gereizten stelle provoziert. Als eine sehr wichtige Unterstützung seiner Theorie führt E. nock an, da $\$$ in dem erythematösen Stadium aller Fälle bei der 
Harnanalyse überaus große Mengen von Indoxyl, Skatoxyl und von Ätherschwefelsäuren gefunden worden sind.

II. Sitzung am 23. September, 9 Uhr vormittags.

Kranken-Demonstrationen im Karlsbader Krankenhause.

Vorsitz: Ledermana (Berlin), fustus (Budapest).

Kreibich (Wien) demonstriert einen Fall von Mycosis fungoides im 2. Stadium der sogenannten flachen Infiltrate vor. Die $28 j$ älurige $\mathbf{P a}$. tientin bemerkt die Hantaffektion seit $6 \mathrm{Jahren}$. Die typische Krankheitserscheinung stellt sich dar als ein rundes, scheibenartiges, entzündliches Infiltrat von bräunlich roter Farbe. Der zentrale Anteil ist eingesunken oder bereits vollständig rückgebildet und mebr gelblich refärbt, während der scharf'e, erhabene, wallartige haud entweder bei stärkerer Irritation leicht nässend ist oder kleienartig abschilfert. An manchen Stellen ist das Infiltrat in zwei konzentrischen Kreisen angeordnet. Das tritt, wenn anch nicht ganz deutlich, an den groben konflu:erten Herden der rechten Brust, sowie um die Gentalgegend hervor. Daneben sieht man auch da und dort in einem Infiltrate fast timmorartige Verdickungen. Ähnliche, Hache, kuchenartige Intiltrate mit zeutraler Rückilildung sind am Rücken lokalisiert, während an den Beurefächen der Extremitäten das bräunlich rote Infiltr.st zum Teile in anaulären, zum Teile in lıalbbogenförmigen Linien augeordnet erscheint. Die Krankheit ist lokalisiert im Gesichte mit ruaden Scheiben an der Stira und um die Mundspalte. Dicht befallen ist ferper die Halshaut, beide Achselhöhleo, Genitalgegend, weniger dicht die Benge- und Streckseiten rer Extremitäten. Unter der Behandlung sind die Infiltrate etwas llacher und breiter geworden, so daB früher nicht konfluierte Herde jetzt konfluieren. Blutbefund normal. In dem scheibenartigen, zentral eingesunkenen und peripher mit einem scharfen Wall umgebenen Irfiltrate liegt das 'Typische dieser Erkrankung und damit auch des Stadiums der Mycosis fungoides. Zu gleicher Zeit sehen wir die Affektion in zwei weiteren Fällen durch ebensolche Krankbeitserscheinungen charakterisiert. In diesen Fällen trat der periphere Infiltrationswall durch zentrale Räckbildung noch deutlicher hervor. Lepra, an welche das Krankheitsbild entfernt erinnert, kann nach der lebhaft roten Farbe, nach dem Fehlen aller übrigen leprösen Erscheinungen und nach dem negativen Blutbefunde ausgeschlossen werden. Die histologische Untersuchung zeigt die typischen, tür Mycosis fungoides beschriebenen Veränderungen.

Diskussion: Delbanco (Hamburg) fragt nach den therapeatischen Bestrebungen bei diesem Fall, ferner ob Riesenzellen gefunden wurden, wie er sie selbst als Fremdkörperriesenzellen mit Einschluß von elastischen Fasern beschrieben hat.

Led ermann (Berlin) weist darauf hin, daß er bereits 1859 typische großkernige Riesenzellen beschrieben hat, die er als Fremdkörperriesenzellen aufgefaßt hat. Der vorgestellte Fall ist durch das lange Vorstadium bemerkenswert. Er selbst hat vor kurzem in der dermatologischen Gesellschaft is Berlin einen Fall demonstriert, in welchem das erythematöse Vorstadium 15 Jahre dauerte. Therapeutisch haben 
große Arsendosen häufig gute Dienste geleistet. Meist verläuft die Krankheit letal.

Kreibich (Wien):

Der zweite demonstrierte Fall ist nur mit einiger Wahrseheinlichkeit als eine prämykotische Erythrodermatitis zu diagnostizieren. Patient, der seit 15 Jahren an der Affektion leidet, zeigt mit Freilassung der Gesicht- und Halshaut fast im ganzen Körper ihrem Alter nach verschiedene Krankheitsherde. Die frischen Herde sind handtellergroße, scharf konturierte, entzündliche Flecke von einer bläulich roten Farbe mit kleinen Hämorrhagien. $\mathrm{Ab}$ und zu sind hellergroBe Herde von dunkler, cyanotischer Farbe, mit zentraler Einsenkung, aber auch diese Herde sind schon aus Konfluenz hervorgegangen und das kleinste Krankheitselement hat das Aussehen eines Lichen ruber planus Knötchen. Solche Knötchen finden sich an der Penishaut, zum 'Teil polygonal begrenzt, mit peripherer Zackenbildung planer Beschaffenheit. Die großen scheibenartigen Herde ändern im Verlaufe ihres Bestandes die Farbe ins bräunlich Rote und hinterlassen endlich ebenso konturierte schmutzig gelbliche Pigmentationen. Die Erkrankung zeigt hie und da Jucken. Atrophien nirgends vorhanden, Mundhöhle, Skrotum frei. Da Lichen ruber planus mit Rücksicht auf die großen, einheitlichen, entzündlichen Flecke aus vielfachen Gründen auszuschließen ist, Pityriasis rubra, circumscripte Hautatrophie, Lepra, ebenfalls ausgeschlossen werden können, so kann die Erkrankung nur in jene Gruppe schwer zu diagnostizierender Dermatitiden eingeteilt werden, die wir Mangels besserer Kenntnisse als Vorstadien der Mycosis fungoides bezeichnen. Knotige Infiltrate bestehen nirgends, obwohl die Affektion 15 Jahre besteht.

Diskussion: Winternitz (Prag): Bezüglich des zweiten Falles möchte W. ebenfalls ein prämykotisches Stadium annehmen. Dagegen möchte er erwägen, ob der erste Fall nicht ein ausgebreitetes Eczema seborrhoicum sei. Der vorhandene seborrhoische Zustand der Kopfhaut, die reichliche Schuppung der Herde, die reine Kreisform einiger Herde bei Fehlen der für Mycosis fungoides charakteristischen nierenförmigen und halbgeschlossenen Herde, das Fehlen von tumorähnlichen Infiltraten scheint gegen Mycosis fungoides und für Eczema seborrhoicum zu sprechen.

Pincus (Berlin) hält den Fall für identisch mit einem vor vier Jahren von Gobert vorgestellten Kranken. Eine Diagnose ward damals nicht gestellt worden, doch bestand eine Neigung, die Affektion als Anfangsstadium der Mycosis fungoides anzusehen. Krankheit.

Saalfeld (Berlin) hält die Affektion für $\bar{D}$ uhring sche

Ledermann (Berlin) spricht sich dagegen aus. Er rechnet den Fall zu den autotoxischen Erythemen und glaubt, daß er dem Typus der Erythéme generalisee exfoliative Besniers einzureihen ist.

Walther Pick (von der Prager Hautklinik) demonstriert einen Fall von sehr ausgedehnter Arsenmelanose bei einem Psoriatiker. Die Melanose besteht seit sechs Wochen und ist nach einer vierwöchentlichen Arsendarreichung in allmählich auf 20 Tropfen Solutio Fowleri steigenden Dosen aufgetreten. Von der intensiven Braunfärbung sind der ganze Stamm und die Extremitäten befallen und an einzelnen Stellen, so in der Schultergegend, an den Seitenteilen des Stammes finden sich bis handtellergroße Herde bestehend aus zahlreichen, kleinsten, nicht über linsen- 
großen weißen Flecken. Von diesen finden sich Übergänge zu solchen mit einer mehr bellroten Färbung, welche neben einer geringen Infiltration auch oberfiächliche Schuppnng erkennea lassen, so daß kein Zweifel dariber besteht, daß es sich um Reste ron Psoriasisefflorescenzen handelt, wenn auch die manifesten Psoriasisherde eine Zusammensetzung aus derartigen kleinsten Effloreszenzen nicht mehr erkennen ließen. In der Medianlinie des harten Gaumens findet sich ein etwa hellergroßer, dunkelbraun pigmentierter Herd.

Diskussion : L ö w en he im (Liegnitz) erwähnt eines Falles, der ohne Arsenmedikation Entfärbung der Haut zeigte, die sich bei späteren Anfällen mit und ohne Arsen nicht wiederholte.

Saalfeld (Berlin) gibt Arsen bei frischem Ausbruch von Psoriasis, da er bei Anwendung von äußeren Mitteln neue Eruptionen auftreten sah.

Ledermann (Berlin) vermeidet bei frischen Eruptionen sowohl Arsen, als Chrysarobin und bedient sich des Wasserstofisuperoxydes (offic.) in Form von Sprays oder einfachen Pinselungen. Obne die geringste Reizung schwinden selbst gröbere Plaques, so daß er die bisher noch nirgends beschriebene Behandlungsform warm empfehlen kann.

Ullmann (Wien): Das Zusammentreffen so prompter Wirkung des Arsen in so geringen Dosen in kurzer Zeit mit intensiver Melanosis ist jedenfalls auffallend. Im allgemeinen wird Arsen viel zu häufig gegeben, weil er doch selten allein zum Ziele führt, ja sehr oft ist er ganz unwirksam.

Pick (Prag) erklärt den Arsenik für ein souveränes Mittel gegen Psoriasis. Er gibt zu, daf Arsen in einzelnen Fallen im Stiche lasse, deshalb könne man jedoch nicht leugnen, daß Arsen in der Mehrzahl der Fälle Glänzendes leiste.

Galewsky (Dresden) schließt sich Ullmann an. Er hält sich nicht für berechtigt, jedem Psoriatiker eo ipso Arsen geben zu müssen, da wir nicht wissen, ob es nicht doch schädliche Folgen hat.

Pick (Prag) tritt dem entgegen, man müsse den Ursachen nachgehen, waram in dev einzelnen Fällen Arsen nichts genützt hat. Im übrigen sah er die beste Wirkung bei der von ihm, wenn nur irgend möglich, bevorzugten Injektionsbehandlung. Iie schädlichen Nebenwirkungen lassen sich bei gehöriger Vorsicht leicht vermeiden.

Ehrmann (Wien) sab Leukoderma bei Braunfärbung der Haut, die von Natur durch Lichtwirkung und Arsen erzeugt war. Der mikroskopische Befund ist wie bei dem Leukoderma syphiliticum. Das Leukoderma bei Chrysarobin beruht auf Ablagerung eines im durchfallenden Lichte bräunlichen, irn auffallenden roten Körpers in der die Plaques umgebenden Hornschicht.

Pick, Walther (von der Prager Hautklinik) demonstriert einen Fall von Atrophia idiopathica cutis. Die Affektion besteht bei dem 21 jähr. Patienten seit 10 Jahren, begann mit einer Abschilferung der Nägel, an welche sich Hautveränderungen anschlossen, die immer das Aussehen der jetzt noch sichtbaren Herde darboten, ohne daß sich je entzündliche

Areh. f. Dermat. u, Syph. Bd. LXVI. 
Veränderungen gezeigt hätten. Eine weitere Veränderung hat mit den Herden nicht stattgehabt, nur an Ausdehnung hat die Affektion allmählich zugenommen, doch ist auch diese seit einem Jahre stationär geblieben. Die Affektion hat hauptsächlich die rechte obere Extremität befallen, nur ganz vereinzelte Herde finden sich über der rechten Schulter und in der rechten Hüftgegend. Am Vorderarme zeigt die Affektion eine deutlich streifenförmige Anordnung; ein etwa $4 \mathrm{~cm}$ breiter, unregelmäßig konturierter Streifen zieht sich vom zweiten und dritten Metacarpophalangealgelenk über den Handrücken und Vorderarm bis ungefähr zur Mitte der Ulva. Außen und innen von diesem Streifen finden sich je ein kürzerer und schmälerer Streifen; außerdem finden sich sowohl am Unter- als auch am Oberarm mit besonderer Beteiligung der Streckseiten unregelmäßig begrenzte Herde von Gulden- bis zu Handtellergröße. Die Haut erscheint an diesen Stellen graublau verfärbt, matt glä́nzend und derart verdünnt, daß die darunter liegenden Gefäße durchscheinen und der palpierende Finger deutlich in eine Vert1efung $z u$ fallen scheint. Die graublaue Verfärbung läßt etwas auf Fingerdruck nach, um einer schwachgelblichen Verfärbung Platz zu machen, am Rande der Herde ist keine Pigmentation sichtbar. Sensibilitätsstörungen oder Parästhesien waren nicht nachweisbar.

Diskussion: Ehrmann (Wien) hält es für eine Entwicklungshemmung, welche sich auf eine Reihe von Hautpartien bezieht und zwar im Bereiche des Bindegewebes und der Papillen.

$\mathrm{Kreibich} \mathrm{(Wien)} \mathrm{ist} \mathrm{jedenfalls} \mathrm{für} \mathrm{die} \mathrm{Diagnose} \mathrm{Hautatrophie,}$ doch ist dieselbe verschieden von der Pospelow schen Form.

Ullmann (Wien) ist derselben Meinung, da die Atrophie schon so frühzeitig eine so intensive Ausbreitung genommen hat und schon von weitem sichtbar ist.

Waelsch (Prag) erwähnt die Halbseitigkeit der Affektion, speziell die bandartige Anordnung an der Streckseite des rechten Armes.

Ledermann (Berlin) verfügt über einen analogen Fall, der vor 35 Jahren begonnen hat. Patient litt zuerst an einer starken Verhärtung der Haut beider Extremitäten bis zu den Nates. Allmählich wurde die Haut der Kniegegend, der Oberschenkel immer dünner und zeigt jetzt eine starke Atrophie mit durchscheinenden Venen, Aufgebobene Hautfalten bleiben bestehen und zeigen den Verlust der normalen Elastizität an. Vor 7 Jahren war die Haut der Unterschenkel bretthart, schlecht verschieblich, wie bei Sklerodermie. Im Laufe der Zeit nimmt auch diese Haut atrophische Beschaffenheit an, wird dünn, leicht verschieblich, so daB sich derselbe $Z$ ustand wie an den Oberschenkeln vorbereitet.

Böhm (Karlsbad) spricht über Keratoma hereditarium palmare et plantare mit Vorstellung eines daran leidenden Kranken. Ausgehend von den über diese Affektion bestehenden differenten Ansichten (Kaposi, Lesser, Joseph, NeiBer u. a. nennen diese Erkrankung Ichthyosis localis palmaris et plantaris, während Unna, Duhring, Neumann hiefür den Ausdruck Keratoma hereditarium palmare et plantare gebrauchen), schildert der Vortragende seinen Fall, der innerhalb drej Generationen bei elf Familienmitgliedern sich fortgeerbt hat. Die Affektion ist an den Handflächen stärker als an den FuBsohlen entwickəlt. Die Haut ist daselbst bedeutend verdickt, durch zahlreiche Falten und Furehen in Plättchen, Platten und Schilder von polygonaler Gestalt und bernsteingelber Farbe geteilt. Die Platten sind an manchen Stellen 
mehrere $m m$ hoch und an den Rändern gerieft. Werden die Hände in aufrechter Stellung gegen das Tageslicht gehalten. sicht man die dicken Hornplatten in ihrer schönen Eigenfarbe. Mäßige Hyperidrosis. Die Beweglichkeit in den Metakarpophalangeal- und Phalangealgelenken ist wesentlich herabgesetzt, das Tastgefühl abgestumpft. Auffallend ist besonders, daß das KältegefähL normal, das Hitzegefühl sehr verlangsamt ist. Die erwähnte Affektion ist nur an den beschriebenen Hautpartien vorhanden nicht wie in den Fällen von $\mathrm{Neumann}$ auch am Dorsum der Handgelenke, Ellbogen und Knie und ist bei allen Familien.zngehörigen in früber Jugend aufgetreten, um bis zum 20. Lebensjahre den Höhepunkt zu erreichen und dann stationär zu bleiben, ohne Tendenz zur Rückbildung.

Diskussion: Joseph (Berlin) hält die Erkrankung für eine während des intrauterinen Lebens eingetretene abortive Ichthyosis.

Weidenfeld (Wien) erinnert daran, daß Kaposi auf zwei Vorgänge nach Hyperidrosis manuum et pedum aufmerksam gemacht hat und zwar Hypertrophie der Hornschicht in den einen Fällen und Atrophie in den anderen. Es mag vun sein, daß wir es hier mit einem habituellen starken Schwitzen zu tun haben, bei dem es zu mächtiger Hornauflagerung gekommen ist.

Voerner (Leipzig) hält den Fall für nicht typisch. Die Hornmassen siad nicht wie beim ausgesprochenen Keratoma anf Hand- und Fußfäche verteilt und begrenzen sich demnach nicht am Rande derselben in typischer Weise. Sie zeigen vielmehr eine herdförmige, stärkere Entwicklung, wie man sie bei anderen Verhornungsanomalien beobachtet (Schwielenbildung und Hyperidrosis u. a.).

Böhm (Karlsbad): Fall zur Diagnose. Ein 27jähriger lediger Mann leidet von Kindheit an an einer Hautafiektion, die symmetrisch an den oberen und unteren Extremitäten sowohl an der Beuge- als Streckscite lokalisiert ist. Die Affektion juckt kolossal Tag und Nacht und läßt nur in protrahiertem Bade und bei künstlicher Schweißerregung etwas nach. Eltern und acht Geschwister leben und sind gesund. Wie Haut der ergriffenen Partien fühlt sich derbe, trocken, rauh und verdickt an. Alienthalben sind zalılreiche stecknadelkopforobe, Hache, glänzende, hornartige Knötchen, die an die Haarfollikel gebunden, zum Teile von einem Haare durchbobrt sind, zum Teile kann man durch Abkratzen der obersten Schichten ein Haar zum Vorschein bringen. An den Tibien ist die Haut bretthart, nur in dicken Falten abhebbar, zahlreiche Kratzeffekte und Borken. Die Haut ist an den erkrankten Stellen braungelb, an deu Unterschenkeln livid. Inguinaldrïsen vergrößert, indolent. Temperatursinn normal, Tast- und Sihmerzgefühl herabgesetzt.

Diskussiou: Kreibich (Wien) hält die Affektion für einen Lichen simplex chronicus.

Joseph (Berlin) glaubt, daß es sich um eine besondere Form von Prurigo handelt. Charakteristisch hiefür sei die vorwiegende Lokalisation an den Streckseiten der Extremitäten. Gegenüber dem Lichen simplex betont er, daß die Affektion nicht als angeboren beschriehen ist.

Ehrmann (Wien) sah denselben Fall vor Monaten, wo zahlreiche Pusteln vorhanden waren. Er muß sich gegen Prurigo aussprechen; schon der scharfe Übergang von erkrankter Haut zur normalen, die follikuläre dichtgedrängte Anordnung spricht entschieden dagegen. Das Aus- 
sehen des Falles, wenn man von der Lokalisation absehen will, spricht für Lichen simplex chronicus Vidal. $\mathrm{Ehrmann}$ stellt übrigens keine Diagnose, sondern er hält die Form für eine follikuläre Erkrankung bisher unbekannter Art.

Pincus (Berlin) näbert sich der Ansicht Kreibichs. Die Affektion ist nicht selten, besteht viele Jahre, bietet stets die Form stark juckender, rauher, follikulärer Knötchen, in Ringen gestellt. Durch die Ähnlichkeit des Verlaufes mit der Prurigo erinnert das Leiden an die eine Abart des Lichen chronicus darstellende Prurigo diathesique. In einem Falle des Beobachters zeigten sich Lichen ruber planus Ringe am Penis. Häufig bestehen dabei urtikarielle Eruptionen, welche durch den Juckreiz die lichenisierte Beschaffenheit der Haut verstärken.

W aelsch (Prag) stimmt mit Joseph darin überein, daß es sich nicht um Lichen simplex chronicus handle, auch als Prurigo kann es nicht anfgefaßt werden. Am besten ist wohl, die Krankheit als follikuläre entaündliche Keratose za bezeichnen.

Weidenfeld (Wien) beobachtete mehrere ähnliche Fälle, die er in der Wiener dermatologischen Gesellschaft vorgestellt hatte.

Delba nco (Hamburg) sieht auch in dem Falle cine besondere follikuläre Affektion, bei der man nicht eine Einfügung in die bekannten Schemata versuchen soll. Er möchte gegen das Jucken die Hypnose vorschlagen.

Prof. Pick (Prag) demonstriert eine Re he interessanter Fälle aus der Prager dermatologischen Klinik, darunter zwei Fälle von Pemphigus, einen Fall von Erythromelie Pick und zwei Fälle von Melanosis lenticularis progressiva Pick (Xeroderma pigmentosum Kaposi) und knüpft an diese Demonstrationen einen Vortrag über die Pathogenese der Prozesse. An diese Demonstrationen schließt sich eine Diskussion, an welcher sich die Herren Justas, Demetriade, Joseph, Ehrmann, Delbanco und der Vortrage nde beteiligen.

Guth (Karlsbad) demonstriert eine Sklerodermie mit Muskelatrophie. Bei einem 8jährigen Mädchen entwickelte sich inuerhalb weniger Wochen im Herbste 1900 eine Sklerodermie. Sie begann an der Brust-und Bauchhaut, ergriff dann die rechte Gesichtshälfte, den rechten Arm und das linke Bein. Jetat besteht eine Sklerodermie der rechten Gesichtshälfte (II. und III. Trigeminusast) an der Beugeseite des rechten Armes, Aulienseite des linken Beines auf den Fubrücken übergreifend, ferner diffuse Herde im atropbischen und Pigmentationsstadium auf Brust und Bauch, an anderen Stellen (Rücken, Glutaei) einzelne sklerotische Plaques. Im Bereiche der rechten Gesichtshälfte führte die Sklerodermie zu einer hochgradigen Atrophie der unterliegenden Weichteile. Am auffallendsten ist die bedeutende Volumsabnahme der Muskulatur des linken Beines, während an der Außenseite desselben nur ein schmaler Streifen von Sclerodermie hinzieht. $Z \mathbf{u}$ dieser Atrophie kam es erst ein Jahr nach Beginn der Krankheit.

Diskussion: Justus (Budapest) erinnert daran, daß Rayna $\mathrm{d}$ s. Krankheit und Sklerodermie häufig koinzidieren.

Galewsky (Dresden) behandelte drei Fälle mit Thiosinamin, wovon einer gekeilt, der zweite gebessert und der dritte fast geheilt ist.

Weidenfeld (Wien): Es ist eine irrige Auffassung, daß die Sklerodermie immer nur die Haut betreffe und dab zum Beispiel die 
Atrophie der Beine auf Druckatrophie zurückzuführen sei. Vielmehr sei der Beginn der sklerodermie oft in dem intermuskulären Bindegewebe zu suchen, und dann ergreift sie erst die Haut.

Pincus (Berlin) hat in einem Falle von Sklerodermie eines Beines die Atrophie der Muskulatur gleichmäßig der zunehmenden Konstriktion des Beines durch Zunahme der Hautstarrheit folgen sehen.

Ehrmann (Wien) hält die Veränderung für eine Sklerose des intermuskulären Bindegewebes, welche nach dem Stadium des Ödems aufgetreten ist.

Guth (Karlsbad): Sklerodermie und Sklerodaktylie.

Bei einem 31jährigen Mädchen traten vor 10 Jahren die Erscheinungen lokaler Synkope an den Fingern beider Hände auf, sobald die Kranke sich kalter Temperatur aussetzte. Drei Jahre später trat das Stadium lokaler Asphyxie auf, in welchem Patientin vorgestellt wurde. Seit fünf Jahren besteht auch Sklerodermie an der Haut der Vorderarme. In letzter Zeit trat auch die maskenartige Veränderung des Gesichtes hinza. An den Endphalangen der Finger finden sich der Fingerkuppe entsprechend etwa linsengroße, unregelmäßige, eingezogene Narben. Die Nägel erscheinen am Rande gerieft, etwas verdickt. Die Haut der Finger fühlt sich ebenfalls verdickt an.

Diskussion: Löwenheim (Liegnitz) erwähnt, daß sich bei einem Kinde, dessen Plaques mit Elektrolyse behandelt worden waren, zugleich die Funktion der atrophischen Muskulatur wesentlich gebessert hat. Der Knabe, bis dahin Linkshänder, wurde wieder Rechtshänder.

III. Sitzung am 23. September, nachmittags $2^{1 / 2}$ Uhr.

Vorsitz: Saalfeld (Berlin), Demetriades (Jassy).

Delbanco (Hamburg) demonstriert 1. ein primäres Urethralcarcinom der pars pendula penis.

Dr. A l s be r g (Hamburg) und Redner haben den Fall bereits in der biologischen Sektion des ärztlichen Vereines in Hamburg vorgestellt.

2. Die wohlgelungene Photographie eines Falles von Scleroderma circumscriptum. Der erythematöse Hof ist sehr gut sichtbar.

Bardach (Kreuznach): Quellsalzseifen bei Hautkrankheiten.

B. bespricht die Anwendung der Kreuznacher Quellsalzseifen, die in verschiedenen Stärken und in Kombination mit Schwefel, Teer, Jod und Bromnatrium hergestellt werden.

Weidenfeld (Wien): Über Verbrennungstod.

Die sebr interessanten Ausführungen Ws. sind bereits als Originalartikel im LXI. Bande des Archiv für Dermatologie und Syphilis erschienen.

Diskussion: Grosz (Wien): erwähnt die grundlegende Arbeit von $R$ ei $B$. Er selbst hat in einer Reihe von letalen Verbrennungen konstant Pyridiu im Harne gefunden (gleich Reiß), dasselbe in einzelnen Fällen durch eine Schmelzpunktbestimmung identifizieren können. Die 
Angaben von Spiegler und Fränkel konnte er nicht regelmäßig bestätigen. Jedenfalls ist der Pyridinbefund von großer Wichtigkeit.

Kreibich (Wien): Über einige melanotische Pigmentationen

K. spricht über die Verschiebung des Pigmentes aus der Cutis in die Epidermis und beweist die dadurch bedingte Pigmentation der letzteren durch Präparate von Schleimhautpigmentationen. Die Pigmentflecke stammen von einem Fall von Sommerprurigo und von einer Arsenmelauose.

Ähnliche Pigmentverschiebungen erfolgen bei Xeroderma pigmentosum bei Carcinom.

Diskussion: E hr mann (Wien) hebt hervor, daß die Frage, ob das Pigment von Hämoglobin stammt, unabhängig ist von der Frage des Einwanderns der an der Mesodermgrenze gebildeten Melanoblasten in die Epidermis, der Abgabe des Pigmentes an die Epidermiszellen. E h. demonstriert zwei Präparate von älteren Katzenembryonen, in denen neben fertigen Haaren junge Haarkeime vorhanden sind. Zwischen Papille und Epithelzapfen sind die Melanoblasten mit Beginn der Verzweigung zu seben. Auch seitswärts sind die Epithelzapfen von Melanoblasten umlagert.

Galewsky (Dresden): Beitrag zur Ätiologie der Akne.

G. spricht von einer "Bäckerakne". Unter 124 hautkranken Bäckern waren 44 mit Akne und 23 mit Eczema seborrhoicum. Alle Akne kranken Bäcker waren anämische, schwächliche, schlechtgenährte Individuen im Pubertätsalter. Die Ursache liegt in der Pubertät, der Anämie und Chlorose der Kranken, dem Mehlstaub und der Backofentemperatur.

Diskussion: D e 1 b a n c o (Hamburg) berichtet über einen Fall von Akne universalis mit Freilassung des Gesichtes und Befallensein der Schamgegend und des behaarten Kopfes. Syphilis.

Breitenstein (Karlsbad): Die Circumcision in der Prophylaxe der

An der Hand einer Statistik gilbt B. seine diesbezüglichen Beobachtungen über die günstigen Wirkungen der Zirkumzision bekannt, die er während seiner zwanzigjährigen ärztlichen Tätigkeit auf Java gemacht hatte.

Löwenheim (Liegnitz): Öber urticarielles Ödem.

Unter diesem Namen beschreibt L. in Niederschlesien häufig vorkommende Schwellungen, die in Begleitung von Urticaria, inneren Erscheinungen, Magen- und Darmerkrankungen und konstanten Neuralgien im Hochsommer gehäuft vorkommen. Sie treten meist akut auf mit intermittierendem Fieber, abendlichen Schüttelfrösten und rezidivieren häufig. Keine Plasmodien. Die Ödeme sind meist im Gesichte in der Nähe der Schleimhäute, ja oft werden die Schleimhäute selbst ergriffen. L. hält die Affektion für eine Infektionskrankheit. In der Literatur sind solche Fälle unter Ödema circumscriptum Quinke, Ödema angioneuroticum Strübing, Hydrops hypostrophos Schlesinger, urticarielles Ekzem Neisser veroffentlicht. Therapeutisch haben sich Chlorkalzium, Salizyl und Chinin bewährt.

Diskussion: P i ck (Prag) erinnert an eine größere Zahl solcher Fälle, die ihm aus Sumpfgegenden (R o s e n b e rger.Teiche) zur Beobachtung kamen. Er faßte dieselben als eine der Malaria verwandte Erkrankung auf. 
E hrmann (Wien) teilt mit, daB er bei mehreren Kranken, die früher Malaria hatten, beobachtete, daß, wenn sie sich mit Lues infizierten, und die Inunktion eingeleitet wurde, neue Malariaanfälle auftraten, so daß die $\mathrm{Hg}$-Kur sistiert und Chinin gereicht werden mußte, bis die Anfälle aufhörten. Milztumor war immer vorhanden.

L ö en h e im (Liegnitz) konnte palpatorisch niemals Milztumor nachweisen, percutorisch sehr selten.

$J$ ustus (Budapest) erwähnt zwei Fälle von Malaria, die mit Lues kompliziert, dieselben Beobachtungen machen ließen, wie Ehrmann erwähnte.

De metriade (Jassy) bespricht das häufige Vorkommen des urticariellen Ödemes bei Kindern in Rumänien. Bezüglich Malaria und Lues erwähnt D., daß die Malaria die Lues verschlimmert.

A. Kraus (aus der Prager Hautklinik): Über Rhinosklerom.

K. bestätigt die von $R$ on a gemachte Beobachtung der metastatischen Lymphdrüsenerkrankung bei Khinosklerom. Es gelang aus der Lymphe einer vergrößerten, regionären Lymphdrüse Kapselbazillen zu isolieren. Histologisch dagegen konnte $K$. im Drïsengewebe die Bazillen nicht auffinden, ebenso wenig charakteristische Zelldegenerationen, Mikuliczsche und hyaline Zellen nachweisen. Es fand sich nur ein subakuter entzündlicher Prozeß im Drüsengewebe ohne jede Spezifität, ebenfalis in Übereinstimmung mit $\mathrm{R}$ ona und $\mathrm{H} u$ ber. Lokale Übertragungsversuche auf Ratten blieben erfolglos, jedoch intraperitoneal injizierte Kulturen erwiesen sich als hochgradig pathogen. Der Bazillus ist in frischen Kulturen Grammbeständig und zeigt bei Nachfärbung mit konzentrierter wässeriger Saffraninlösung eine gute Kapselfärbung.

A. Kraus (ans der Prager Hautklinik): Über Knotenbildung in der Haut durch circumscripte entzündliche Atrophie des subkutanen Fettgewebes.

Bei einem 15jährigen Mädchen mit Pemphigus und hämorrhagischer Diathese traten zeitweise eigentümliche Knoten in der Haut der Extremitäten auf, welche bis erbsengroß, derb, in den tieferen Hautschichten saßen, manchmal schmerzhaft wurden und eine livide Verfärbung der Haut zeigten. An den oberen Extremitäten ließen sich ferner in der Tiefe der Haut gelegene Stränge tasten, die stellenweise aus perlschnurartig aneinander gereihten Knotenbildungen zu bestehen schienen. Zur Zeit des Auftretens der Knoten und Stränge war das Allgemeinbefinden sehr gestört, der Frnährungszustand sehr schlecht.

Während $1^{3 / 4}$ Jahren bildeten sich diese Hautveränderungen zum geringsten Teile zurück, jedoch ohne wahrnehmbare Residuen oder Atrophie zurückzulassen. Die bistologische Untersuchung ergab einen im subkutanen Fettgewebe lokalisierten Entzündungsprozeß, der mit Atrophie des Fettgewebes einherging. Fin zweiter ähnlicher Fall, der aber nicht histologisch untersucht wurde, betraf ein drei Wochen altes Kind, bei dem mit dem Auftreten schwerster dyspeptischer Störungen in den tieferen Schichten der Haut des Stammes und der Extremitäten multiple derbe Knoten sich bildeten, welche bei zunehmender Finnährung innerhalb eines Monates wieder schwanden. K. ist der Ansicht, daB der erwähnte Prozeß als ein Symptom der die Kräfte des Gesamtorganismus konsumierenden schweren Allgemeinerkrankungen aufzufassen sei.

A. Kraus (aus der Prager Hautklinik): Beiträge zur Kenntnis der Alopecia congenita familiaris. 
Vier Kinder gesunder Eltern waren mit normaler Behaarung geboren. Einige Wochen nach der Geburt gingen die Haare wieder vollständig verloren, ohne später wieder nachzuwachsen. Die vorliegende Hemmungsbildung ist auf eine Störung des fötalen Haarwechsels zurückzuführen. Ursache ist unbekannt. Durch die bi-tologische Untersuchung der Kopfhaut konnte das Vorhandensein von Haaranlagen nachgewiesen werden, doch zeigten dieselben hochgradige regressive Veränderungen. Ein so ausgesprochener familiärer Charakter der Alopecia congenita, die bier sämtliche Kinder einer Familie betraf. ist bisher nie beobachtet worden.

Diskussion: L e d e r m a n $\mathrm{n}$ (Berlin): $\mathrm{P}$ i n c u s veröffentlichte einen analogen Fall von Alopecia congenita totalis bei Vater und Sohn, bei denen es nur zu einer dünnen Fläumchenbildung gekommen ist. Außerdem berichtet er von einem periodischen Haarwechsel bei einem 20jährigen Mädchen. gewebe.

Hasler (aus der Prager Hautklinik): Zelleinschlüsse im Lupus-

Die zuerst von $\mathrm{L}$ ang beschriebenen Zelleinschlüsse, die sich in Hs-Fall (Lupus von Ohrläppehen) an die überaus reichlich vorbandenen Riesenzellen gebunden zeigten, erwiesen sich bei der histologischen Untersuchung weder als eine Art der verschiedenen bekannten Zelldegenerationsformen noch beim mikrochemischen Nachweise als aus Kalksali en bestehend. H. glaubt, daß es sich um Degenerationsvorgänge der Riesenzellen selbst handle, doch läßt er die Frage, welcher Natur dieselben seien, offen.

Diskussion: P in cus (Berlin) hält die Riesenzellen für Fremdkörperriesenzellen.

D e 1 b a n c o (Hamburg) ist derselben Ansicht. Die Annahme, daß die Gebilde verändertes Protoplasma von Riesenzellen seien, ist nach seiner Ansicht durchaus abzulehnen. Ihm ist es nicht gelungen, die Gebilde aufzulösen.

Brat (Berlin): Die Hautempfindungen nach endermatischer Anwendung einiger neuer Medikamente.

Die Notwendigkeit, schmerz- und juckenbeseitigende Mittel bei Hautkrankheiten anzuwenden, führte zum Gebrauche von Cocain, Orthoform, Bromocoll. Durch Anwendung einer Salbengrundlage (Resorbin, Lanolin) wird der Leitungswiderstand der Haut vermindert. Nach Anwendung von Orthoform- und Bromocollsalben wurde eine Herabsetzung für Druck und den faradischen Strom experimentell nachgewiesen. Erkrankte Haut, Kontinuitätstrennungen ermöglichen die Resorption von Salben; wenn Bromocoll resorbiert wird, müßte Tannin und Brom im Harne sich nachweisen lassen. Der Bromnachweis gelang. Durch die änßere Anwendung von Bromocoll werden die Nervenendigungen in ihrer Erregharkeit herabgesetzt.

An der Diskusion beteiligten sich die Herren Waels ch (Prag), Ehrmann (Wien), J o seph und Brat (Berlin). 
IV. Sitzung am 24. September, 3Uhr nachmittags.

Vorsitz: Max Joseph (Berlin), Kreibich (Wien).

Schanz (Dresden): Die Augenentzündungen der Neugeborenen und der Gonokokkus. ( $Z u$ diesem Vortrage war die Sektion für Augenheilkunde eingeladen).

1. Fis gibt Augenentzündungen der Neugeborenen, welche unter dem Bilde der vollkommen typischen Blennorrhoe verlaufen und bei denen trotz sorgfältigster Untersuchung der Gonokokkus nie gefunden wird.

2. Auber dem Gonokokkus soll nocb eine ganze Reibe anderer Microorganismen im stande sein, das typische Bild der Ophtalmoblennorrhoe der Neugeborenen hervorzurufen.

Das sind die wichtigsten Ergebnisse der in den letzten Jabren vorgenommenen diesbezüglichen Untersuchungen. Dies steht im Widerspruche mit dem Lehrsatze, daß der $N$ e is s e r sche Gonokokkus der alleinige Erreger der akuten Ophthalmoblennorrhoe der Neugehorenen sei. Untersuchungen von $\mathrm{Seh}$, $\mathrm{Kr}$ on e $\mathrm{r}$ und $G$ roen ow bewiesen, daß die Augenentzündungen der Neugeborenen ätiologisch keine einheitliche Erkrankung sei, daß die Bindehaut der Neugeborenen auf eine große Anzahl von Bakterien in gleicher Weise reagiere.

Diskussion: Uhth of $f$ (Breslau) stimmt diesen Anschaungen nicht zu. Nach seiner Überzeugung bieten die Gro e n o v schen Untersuchungsresultate eine Stütze für die allgemein gültigen Anschauungen über die Rolle der Gonokokken bei der Conjunctivitis blennorrhoica im Sinne Neissers. Die Frage der sogenannten Pseudagonokokken hält er noch für eine of?ene.

Galewsky (Dresden) ist der Ansicht, dalb die typische Augenblenvorrhoe durch den Gonokokkus hervorgerufen wird. Sowie es aber eine nicht gonorrhoische Urethritis gibt, wird es auch ebensolche Augenblennorrhoen ohne Gonokokken geben.

Goldberg (Wildungen) hat eine akute primäre Streptokokken Urethritis gesehen.

Grosz (Wien) verweist auf $\mathrm{Hellera}$ Arbeit und seine mit Kra us gemeinsame Untersuchung. Es gibt außer der Conjunctivitis gonorrhoica auch solche durch andere Mikroorganismen hervorgerufene.

$\mathrm{Schanz}$ (Schlußwort) bat sich absichtlich jeder eigenen Meinungsänßerung enthalten und nur die Thesen von Finger und Groenow zitiert.

Joseph und Piorkowski (Berlin): Weitere Beiträge zur Lehre von den Syphilisbazillen.

Ausgehend von der Überlegung, daß in dem Sperma überhaupt und vielleicht noch viel länger wie in anderen Körpersäften das luetische Virus sich aufhalte, daß durch dasselbe allein eine Infektion bervorgerufen werden könne, gingen J. und $P_{c}$ an die Untersuchung des Samens und Kultivierung des frischen Ejaculates auf einer normalen sterilen Placenta. Auf den mit syphilitischem Sperma infizierten Placenten fanden sich schon nach 24-48 Stunden kleine, tautröpfchenartige Kolonien, die allmählich einen grauen Farbenton annahmen und dann mehr oder 
minder konfluierten. Durch das Färbeverfahren wurden nur Stäbchen nachgewiesen, welche plump, an einem Ende kolbig verdickt, sehr häufig körnerartig degeneriert waren und eine staketenartige Lage aufwiesen. Die Stäbchen waren 4-8 $\mu$ lang und $0 \cdot 2-0 \cdot 3 \mu$ dick, und hatten die Form von Diphtheriebazillen. Deutliche Färbbarkeit mit verdünntem Karbolfuchsin oder Gentianaviolett. Nach Gramm waren sie positiv. Deutliche Polfärbung. Sie ließen sich nur durch Zwischenschaltung der Placenta weiter kultivieren. Ihre Züchtung gelang am besten auf menschlichem Blutserum. So glückte der Nachweis dieser Bazillen bisher in 39 Fällen frischer Lues innerhalb der Zeitdauer von zwei Monaten bis zu zwei Jahren post infectionem. Bei 15 Fällen von Spätsyphilis konnten die Bazillen nicht gefunden werden.

Der Nachweis der beschriebenen Bazillen gelang auch im Sekrete des harten Schankers, in Papeln, Plaques muqueuses, Condylomata lata und in zwei luetischen Inguinaldrüsen. Überimpfungen von Kulturen dieser Bazillen auf Mäuse, Meerschweinchen und Kaninchen, sowohl subkutan als intraperitoneal vorgenommen, hatten keinen Einfluß auf die Tiere erzeugt. Nur bei einem Schweine trat hernach ein den Tierärzten auffälliges, aber unbekanntes Exanthem auf. Es ist zu erwäbnen, daß stets sorgfältige Kontrollversuche angestellt wurden. P. ist es gelungen, durch ein besonderes Färbeverfahren in dem frischen, syphilitischen Spermaejakulat die gleichen Bazillen darzustellen. Außerdem gelang auch der Nachweis in der staketenartigen Lagerung in den Lymphräumen von luetischen Drüsen. J. hält diese Bazillen mit großer Wahrscheinlichkeit für die Erreger der Syphilis.

Piorkowski (Berlin): bespricht des Genaneren die von ihm und J o s e ph gemachten Versuche, wiederholt nochmals die von J os e ph gegebene Beschreibung der gefundenen Bazillen mit besonderer Betonung der guten Darstellungsfähigkeit der Babes-Ernst schen Körperchen. Im hängenden Tropfen findet sehr bald eine Agglutination statt, während, nur wenige der Bakterien frei im Gesichtsfelde oszillierend bleiben.

Diskussion: Waelsch (Prag): Es wäre viel einfacher gewesen, statt aus dem Sperma die Bazillen aus dem Blute zu züchten. Der von J. und P. gefundene Bazillus, der nach seinen Eigentümlichkeiten des Wachstums, Form, Färbbarkeit ein Pseudodiphtheriebazillus ist, ist mit dem von van $\mathrm{N}$ i e s s e n zu identifizieren. Nach seinen eigenen Untersuchungen, die noch nicht ganz abgeschlossen sind, könne er die Ergebnisse der Niessen schen Untersuchungen zum Teile bestätigen. Die Untersuchungen erstrecken sich auf 10 Fälle sekundärer Lues, 2 Fälle von Lues gummosa, 1 Fall von zerfallenem Gumma, 2 Fälle von Ekzem und Psoriasis. In 2 Fällen von florider sekundärer Lues wurden inguinale Drüsen exstirpiert und untersucht. Die: Untersuchung wurde in der Weise vorgenommen, daß mit steriler Spritze aus der Vena mediana Blut entnommen und in verflüssigte Gelatine gebracht wurde. Von den 10 Fällen sekundärer Lues konnte $\mathrm{W}$. in 6 den Niessen'schen Bazillus in Reinkultur darstellen, in 3 bekam er einen großen Diplokokkus in Reinkultur, in 1 Fall nichts. Aus den Drüsen wuchs der Bazillus beidemal in Reinkultur; bei tertiärer Lues und bei Gesunden war das Resultat negativ.

W. beschreibt nun ausführlich den gefundenen Bazillus, dessen Färbbarkeit, Wachstum auf den verschiedenen Nährböden und die Resultate der mit Reinkulturen vorgenommenen Impfungen. Impfungen 
wurden an drei Schweinen, Meerschweinchen und Kaninchen vorgenommen, welche bei den beiden letzteren ein negatives Resultat ergaben. Bei den Schweinen hingegen gelang es, durch Impfungen mit den Bazillenreinkulturen Exantheme zu erzeugen, welche analog waren denen, die van Niessen beobachtet und beschrieben und die anch Adrian, Hügel und Holzhäuser durch Verimpfung von Blut Syphilitischer, Sklerosenreste u. s. w. bei Schweinen hervorrufen konnten. Die Gleichartigkeit dieser Erscheinungen, das Auftreten nach zirka 7wöchentlichem Inkubationsstadium, die Recidive in kleineren und größeren, Pausen, endlich die Ábnlicbkeit der den Tierärzten unbekannten Exantheme mit den Effloreszenzen der Syphilis beim Menscben sind sicher sehr auffallende Momente. Die histologische Untersuchung eines mit Exanthem bedeckten Hautstückes, das excidiert wurde, ist noch nicht abgeschlossen. Der gefundene Bazillus wurde auch auf seine Agglutinationsfäbigkeit durch das Serum Syphilitischer geprüft und bestätigt.

Das häufige Vorkommen des beschriebenen Bazillus im Blute Syphilitischer und die Möglichkeit der Hervorrufung einer den Tierärzten nicht als Schweinekrankheit bekannten Erkrankung durch Infektion der Schweine mit dessen Reinkulturen, ist gewiß eine auffallende Erscheinung. Weitere Versuche werden erst feststellen, ob der Bazillus in ätiologische Beziebung zur Lues der Menschen gebracht werden dürfe oder nicht.

Winternitz (Prag) hat mit Kral Sekret von gereinigten Sklerosen untersucht and Ansammlungen von Mikroorganismen gefunden welche denen von Joseph und Waelsch gleichen. Weiter wurden Kultureu vom Blute Luetischer (1. und 2. Exanthem) aut Menschenblutserumagar angelegt und nach 10 Tagen wurde das Auswachsen von kleinen, sehr dicht stehenden, gelbbraun erscheinenden, runden, respektive polyedrischen Kolonien gesehen, die sich als aus polgefärbten, kurzen Bazillen zusarnmengesetzt darstellten. Über Färlungen und Tierimpfungen könne er noch nicht berichten, doch werden solche vorgenommen.

Kreibich (Wien) fragt an, ob bei der Grammbeständigkeit des Bazillus derselbe nach dieser Färbung im Sperma oder nach der W e ig e rtschen Färbung im Schnitte nachweisbar ist. Er hatte dabei kein Resultat.

Delbanco (Hamburg): Löffler hat bereits bei Condylomata lata Psendodiphtheriebazillen nachgewiesen.

Vorderhand mul von Begleitbakterien bei der Syphilis gesprochen werden. Er selbst legt den Schnittpräparaten der frisch extirpierten Lymphdrüse keinen besonderen Wert bei; die Exantheme bei den Tieren können vielleicht septische Exantheme sein.

Pick (Prag) ist erfreut, daß die Vortragenden sich jeglicher Schlüsse enthalten und nur die tatsächlichen Resultate ibrer Untersuchungen mitgeteilt baben; er wünscht, daß man es vermeide, die gefundenen Bazillen als Syphilisbazillen zu bezeichnen und nur von Bazillenbefunden bei Syphilis spreche. 
Jos eph (Berlin) freut sich über die im wesentlichev übereinstimmenden morphologischen und kulturellen Ergebnisse hinsichtlich des Bazillus. Die Hauptsacbe ist, da $B$ von vielen Seiten darüber gearbeitet wird; man müsse allerdings abwarten, ob man es nur mit Bazillen bei Lues oder mit Bazillen der Lues zu tun haben werde.

Pi orkowski (Schlußwort): Die Bazillen wurden auch im Blute nachgewiesen, das aus einer Armvene entncmmen wurde. Desgleichen gelang es, die Bazillen im Schnitte nach dem Weigertschen Verfahren unter Anwendung der besprochenen Modifikation darzustellen.

\section{Ehrmann (Wien): Demonstration von Injektionspräparaten.}

Zum Studium der Frage, ob die Stränge, welche man bei Initialsklerose unter der Haut des Membrum findet, von Blut- oder LymphgefäBen ausgehen, hat Ehrmann unter Anwendung eines besonderen Injektionsverfahrens 21 amputierte Präputien untersucht. Das hervorstechendste Merkmal der frischen Infiltration ist eine Vermehrung der Blutgefäße durch Neubildung von Kapillaren. Diese Vermehrung betrifft nicht bloß das Massiv der Sklerose, sondern setzt sich auch streifenförmig in die Umgebung fort, so daß von den Gefäßen, welche z. B. die glatten Muskelfasern, die Nerven und mit ihnen die Pacchinischen Körperchen begleiten, sich ein ungemein reiches, selbständig erscheinendes Netz von Blutkapillaren bildet. Aber auch in den Infiltrationsstreifen, welche bloße Gewebsspalten begleiten, entsteht ein solches Netz von Blutgefäßen mit neuen Kapillaren und Schlingen in der Peripherie. Gegen den erodierten Teil der Sklerose nehmen die Kapillarschlingen der Papillen, sowie die Papillen selust an Mächtigkeit zu; selbst an der Grenze des erodierten Teiles, wo es keine Papillen mehr gibt, ist noch ein reiches Kapillarnetz vorhanden, um dann allmählich abzunehmen. Die von der Tiefe kommenden Kapillaren sind durch das dichte Infiltrat komprimiert, während das oberfäachliche Netz erweitert und der Belag durch Hämoglobin grünlich gefärbt ist. In der Mitte des erodierten Teiles selbst sind die Gêfäße spärlich, sie sind größtenteils zu Grunde gegangen. Bezüglich der Lymphgefäße ist zu erwähnen, daß dieselben im nicht erodierten Massiv der Sklerose mit den Blutkapillaren sich durchflechten; eine Sonderstellung bekommen sie erst in der subpapillären Schichte. Von hier aus sind sie der Grundstock, um den sich die Infiltratmasse lagert. Nach dem Fortschreiten der Gefäßneubildung und nach deren Beziehungen zu den Lymphgefäßen und Lymphspalten läßt sich folgendes schließen. Das in die Haut gelangte syphllitische Kontagium gelangt zunächst in die Gewebsspalten, kann schon von hier aus auf die Blutgefäle wirken, indem es auf chemotaktischem Wege Leukocyteneinwanderung und Gefäßneubildung erzeugt. Im weiteren Verlaufe gelangt es in die Lymphgefäßse, wirkt hier in derselben Weise auf die subendothelialen, sowie auf die äußeren Blutgefäße und erzeugt hier ein inneres Infiltrat. Das äußere Infiltrat kann teils durch die den größeren Lymphgefäßen zuströmenden Lymphkapillaren erzengt werden - führt stellenweise zur Verengerung oder Obliteration des Lumens, und erzeugt Lymphocyteninfarkte in den Blutgefäßen.

Ehrmann (Wien): Über die Sklerosenreste und ihre Bedeutung.

Durch klinische Erfahrung kam Ehrmann zur Überzeugung, daß in jenen Fällen, in denen die Sklerosen nach Schluß der Behandlung noch nicht geheilt waren, Syphilisrezidiven viel häufiger waren als in anderen, und da $B$ in einer Reihe von Fällen aus einem kleinen sklerosenrest eine typische, nlzerierte, manchmal auch mehrfache frische Sklerosen aus der Induration entstanden sind. Ehrmann hat deswegen in 2 Fällen Präputien mit 
solchen Sklerosenresten amputiert, mit Berlinerblau injiziert und untersucht. Es zeigte sich, daß nach 7 Monaten im Narbengewebe an den Lymphgefäßen kleine Anhäufungen von Lymphocyten zurïckgeblieben sind, offenbar als Rest der knotigen Anschwellungen. Als Zeichen, daß nengebildete Blutgefäbe zu Grunde gegangen waren, fand man reitblich Hämosiderin in den Knoten. Es ist nun anzunehmen, daB von diesen lymphangitischen Herden neue Massen von Kontagium noch in die LymphgefäBe und in die Blutbahngelangen, neue Eruptionen veranlassen, sowie auch, daß das in ihnen verbliebene Virus unter günstigen Umständen neue Infiltrationen um die Lymphgefäße setzt und auf diese Weise Reindurationen verursacht. Diese Infiltrate sind aber auch die Ursache der lokalen Rezidiven und deren sind zweierlei: Die sklerosenähnlichen Rezidiven und die in Papelform und später als Gummen. Die papulösen syphiide gehen bekanntlich von den Blutgefäßen aus. Maa muß annehmen, daß in jenen Fällen, wo lange vor Ausbruch des ersten Exanthemes papulöse Fxantheme ad genitale entstehev, das Kontagium aus den Lymphspalten in die benachbrrten Arterjenstämme übergeht. In Beziehung auf die Lymphgefäße kann Ehrmann über eine Reibe von Tatsachen berichten, daB die beschriebenen Infiltratreste an den LymphgefäBen auch der Ausgangspunkt für spätere Gummen sind, besonders bei Gummen des Genitale. Eine Tatsache, welche für Lymphgefäßveränderungen in den Lymphdrüsen spricht, ist die, daß Ehrmann nach Ablauf der Sklerosen in den oberflächlichen Lymphbahnen der Lymphdrüse äbnliche Lymphgefäßinfarkte fand wie in der Initialsklerose.

thritiden.

Galewski (Dresden): Über chronische, nicht gonorrhoische Ure-

Wa 1 lsch setzte bei diesen Erkrankungen folgende Punkte auf:

1. das lange Inkubationsstadium,

2. den von Anfang an chromischen Verlauf,

3. die geringen objektiven Symptome,

4. den stets negativen Gonokokken-Befund,

5. den langwierigen Verlauf,

6. die schlechte Prognose.

G. berichtet über 7 einschlägige, einwandfreie Fälle aus seiner Praxis mit einer Inkubationszeit von 9-14 Tagen, geringen subjektiven und objektiven Beschwerden, geringem schleimig-eitrigem Sekrete. Mikroskopisch und kulturell waren nie Gonokokken nachweisbar, nie Prostatitis oder eine Komplikation von Seite der Urethra posterior.

Sehr widerstandsfähig gcgen jede Therapie.

Diskussion: Winternitz (Prag) hat solche Fälle oft gesehen. Thre Dauer ist sehr verschieden, von wenigen Wochen bis Monaten und Jahren. Bei einem jetzt in Behandlung stehenden Falle besteht eine Prostatitis.

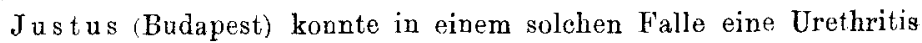
posterior haemorrhagica konstatieren.

Böhm (Karlsbad) beobachtete eine nicht gonorrhoische Urethritis mit beiderseitiger akuter, katarrhalischer Epididymitis, die nicht zur Sterilität geführt batte

Merk, Demetriades, Reis ner erwähnten ebenfalls von solchen nicht gonorrhoischen Urethritiden.

V. Bandler (Prag): Über Cystitis tuberculosa.

Die primäre Blasentuberkulose wurde vielfach angezweifelt und erst Casper hat in letzter Zeit mebrere Fälle mitgeteilt und speziell auf die Gonorrhoe als atiologisches Moment der primären Blasentuberkulose 
hingewiesen. B. wendet sich gegen die Ansicht, daß die Früh-Diagnose so schwierig sei; ihm war es gelungen, schon in den ersten Stadien bei leicht diffus getrübtem Harne im Sedimente die gehäuft liegenden zerfaserten Tuberkelbazillen nachzuweisen. Schwerer nachweisbar wurden dieselben in den späteren Stadien bei zunehmendem Eitergehalt. Unter den Symptomen sind besonders bemerkenswert der grobe Harnzwang (infolge der geringen Kapazität der Blase), der die exzessiven Schmerzen verursacht wie bei keinem anderen Blasenleiden, ferner die oft in bedrohlicher Weise auftretenden kolossalen Blutungen. B. berichtet von einem Falle, in welchem nach $1^{1} / 2$ jährigem Bestande eine ascendierende Nierentuberkulose hinzukam. Nach zweijähriger Daver kam es zur Sectio alta, die von solchem Frfolge war, daß Patient sich in kurzer Zeit erholte und nach 4 Wochen von Schmerzen befreit, das Bett verlassen konnte. Auch die Nierentuberkulose war ganz zum Stillstande gekommen, so daß der Kranke nach 1 Jahre wieder arbeitsfähig geworden war. Dieser Fall beweist, daß die Prognose selbst in den schwersten Fällen nicht aussichtslos ist und einen chirurgischen Eingriff rechtfertigt.

Diskussion: G oldberg (Wildungen): Sterilität sauren, eitrigen Urins spricht für Tuberkulose, falls Gonorrhoe ausgeschlossen werden kann. Sekundärinfektionen sind bei Urogenitaltuberkulose meist artifiziell. Primäre hämatogene Blasentuberkulose ist sehr selten.

Grosz (Wien): Über eine seltene Komplika'ion der chronischen Gonorrhoe nebst Beiträgen zur pathologischen Anatomie der männlichen Urethra.

Bei Untersuchung eines seit $1^{1} / 2$ Jahren an cbronischer Gonorrhoe leidenden Mannes fand $G$. an der Unterseite der Urethra hinter dem Sulcus coronarius ein über erbsengroßes Knötchen, das der Urethra fest und unverschieblich aufsaß. Ab und zu waren Größeuveränderungen an dem Knötchen sichtbar. Ls wurde operativ entfernt und die histologische Untersuchung ergab, daß es aus vielfach gebuchteten und verzweigten Gängen bestand, die sich zu einem kurzen, in die Urethra mündenden Gange vereinigten. Die Gänge selbst entsprechen den Morgagnischen Lacunen. Das eigentümliche Auswachsen derselben und ihre vielfache Verzweigung ist entweder angeboren oder vielleicht durch die Entzündung bedingt. Knötchenartige Bildungen an der Urethra kommen im Verlaufe einer Gonorrhoe nicht selten vor als paraurethrale Entzündungen, Follikel, als Periurethritis. Mit keiner dieser Affektionen kann die vorstehende Beobachtung identifiziert werden.

Benario (Frankfurt a. M.) demonstriert zur Behandlung der Gonorrhoe einen einfachen Apparat "Urosanol", welcher aus einer kleinen Glastube besteht, die mit Protargolgelatine gefüllt ist und auf welche eine Gummiolive oder ein kleiner Nelatonkatheter aufgesetzt wird.

Vorteile: Einfachheit, Reinlichkeit und Handlichkeit.

Dommer (Dresden): Zur instrumentellen Behandlung der gonorrhoischen Striktur.

In den meisten Fällen handelt es sich um eine Verengerung des Lumens. Diese za beseitigen, versagen oft die Dilatatoren. Man ist gezwungen, die Urethrotomia interna auszuführen. Für weite Strikturen eignet sich das Kollmannsche Urethrotom. Zur Bebandlung enger Strikturen schneidet nun D. nicht in einer vertikalen, sondern noch in zwei schrägen Richtungen ein. Dadurch entsteht eine Zerklüftung der Striktur; die einzelnen Taschen hängen in das Lumen des Tubus hinein und werden mit einem von D. konstruierten Instrumente abgetragen. 
Der Apparat kann auch benützt werden zum Abtragen randständiger Papillome.

Goldberg (Wildungen): Die Verhütung der Harninfektion.

I. Die Erforschung der Ursachen der externen Harnintektion führt zu der Erkenntnis, daß Fälle vermeidbar ist.

2. Fernbaltung der Infektionsstoffe, mechanische Entfernung derselben, Anwendung keimtötender und entwicklungshemmender Mittel soll sich auf den Operateur, auf die Objekte der Umgebung, auf die einzuführenden Instrumente und Medikamente erstrecken. Instrumente und Medikamente sind, wo nur möglich, mit physikalischen Methoden keimtrei zu machen.

3. Die Urethralflora hindert eine wirksame Verhütung der Harninfektion keineswegs.

4. Fine intravesikale prophylaktische Desinfektion ist vorzunehmen a) bei allen Fingriffen mit nicht exakt sterilisierbaren Instrumenten (optische, Konduktiv, Verweil-Instrumenten), b) bei Erkrankungen der Vorräume.

5. Die Tatsache, daß in vielen Fällen der Mikrobenimport nicht zur Infektion führt, darf nicht Anlaß werden, nicht allgemein, sondern nur bei den disponierten Fällen aseptiscb respektive antiseptisch vorzugehen; denn wir können nicht immer vorher wissen, ob die Empfänglichkeit für Infektion besteht oder nicht. Wohl aber soll diese Existenz „disponierter Fälle“ Anlaß3 sein: a) Schlüsse über Erfolge von Sterilisatiousmethoden sind nur aus disponierten Fällen zu ziehen. b) Bei diesen Fällen ist die äußere Antiepsis durch die interne prophylaktische I)esinfektion mittelst Verabreichung von Urotropin (2.-3. pro die), Chinin (mehreremale 025) Salol (große Dosen) zu ergänzen; ersetzen kann die interne Harnantisepsis die äußere niemals.

Winternitz (Prag): Über die Wirkung der Balsamica.

Die diesbeziigliche Arbeit erscheint in diesem Archiv.

V. Sitzungam 25. September, 3 Uhr nachmittags.

Vorsitz: Galewsky (Dresden), Merk (Graz), Delbanco (Hamburg).

Freund (Wien): Neuere Erfahrungen über die physikalische Behandlung von Hautaffektionen.

In Paris wurden in jüngerer Zeit zwei Instrumente erfunden, das Radiochromometer und das Spintometer, die jedoch nach Untersuchungen von F. keine besonders exakten Resultate liefern. Ein viel rationelleres I'rinzip zur Bestimmnng der Intensität der von der Vakuumröhre ausgehenden Strablungen liegt dem Hol zknechtschen Chromoradiometer z.n Grunde. Derselbe therapeutische Fiffekt läßt sich mit starker and schwacher Bestrahlung, $d, b$. mit weichen und harten Röluren, bei großen und kleinen Röhrenabständen, bei langen und kurzen Expositionen bei entsprechender Dosierung erzielen. Da die X-Strahlen nur bis zu einer gewissen Tiefe wirken, mulj man, um mehr in die Tiefe wirken zu können, Strahlen von gröberer Wellenlänge (blau-rot) anwenden. Es ist dies die Finsenbestrahlung. Die diesen St rahlen nachgerühmte bakterizide Wirkung ist nach F. nicht vorhanden. Nach Versuchen von F. hat es sich gezeigt, daß die langwelligen Wärmestrablen günstige Wirkungen bei Acne vulgaris; Ulcus cruris haben. 
An der Diskussion beteiligen sich Ehrmann, Euler gegen Rolle und Holzknecht (Wien).

\section{Holzknecht (Wien): Das Chromoradiometer.}

$H$. ist es gelungen, die absorbierte Lichtmenge direkt zu messen. Das Problem der direkten Dosierung des Röntgenlichtes durch direkte Messung der absorbierten Lichtmenge berubt auf der Beobachtung, daß die Färbung durchsichtiger Reagenskörper von der absorbierten Lichtmenge abhänge. Er legt zu diesem $Z$ wecke neben oder auf das Bestrahlungsfeld einen Reagenskörper und achtet bloß darauf, ob der Reagenskörper schon den nötjgen Färbungsgrad erreicht hat. Dieser wird dann an einer kalorimetrischen Skala abgelesen. Die Einheit hat. H. mit s e benannt. Die Vorteile seiner Methode sind leichte Erlernbarkeit und Ausführbarkeit, die zahlenmäfige Exaktheit, die Vergleichbarkeit der literarischen Angaben und der eigenen Resultate. Das gilt auch von der Becquerelbestrahlung.

An der Diskussion beteiligten sich Merk (Graz), Strebel (München) und Holzknecht.

Strebel (München): Lichttherapeutische Spezialitäten und neue Lichtgeneratoren. Beitrag zur Theorie und Praxis der Röntgen-Therapie.

St. spricht zunächst über die Resultate der Behandlung der chronischen Gonorrhoe mit dem von ihm im Vorjahre veröffentlichten elektrischen Glimmlicht, das in sondenartigem Instrument erzeugt wird. Weiterhin ist St. durch Verwendung seiner neuesten Lampe im stande, in der Urethra Lichtreaktion hervorzurufen, welche sich voraussichtlich zur Behandlung der akuten Gonorrhoe verwenden lassen werde. Das Licht der Lampe ist reich an Ultraviolett, enthält aber auch viel Strahlen in Blau und Violett. Der Redner erklärt, daß die Heilung der akuten Gonorrhoe durch das Licht noch Problem ist, daß aber durch die Versuche die Frage in einen diskutablen Standpunkt gekommen sei.

Bezüglich der Röntgen-Therapie fragt es sich, ob der photochemische Effekt der Röntgenstrahlen mit der therapeutischen Wirksamkeit derselben identifiziert werden kann. Die heftigsten photochemischen Wirkungen werden durch Ultraviolett hervorgebracht, das aber schon in den obersten Hautschichten absorbiert wird, also ungenügende Tiefenwirkung entfaltet. Es geht also photochemische Leistung und Heileffekt nicht Hand in Hand. Zur Dosierung der Strahlungen des weichen, therapeutisch wirksamen Rohres wendet er den "Strahlendämpfer" an. S. betont seine Priorität bezüglich der Feststellung einer bakterientötenden Kraft des Radium. Seine Erfolge der Röntgentherapie decken sich mit den bisherigen Erfahrungen. wirksam.

Diskussion: Freund (Wien) hält harte Röhren für unzweifelhaft

Holzknecht (Wien) glaubt nicht mehr recht an Heilungen von Lupusfällen. Die intumeszierten Formen gehen rasch ins Niveau zurück, dann sind sie aber nicht mehr vom Fleck zu bringen. Finsenbehandlung bingegen scheint wirkliche Heilungen gu bewirken.

Ehrmann (Wien) bemerkt zur Photographie eines durch Röntgen geheilten Falles von Alopecia areata, daß solche Fälle auch nach Faradisation und hautreizenden Mitteln heilen, vorausgesetzt, daß die Papillen nicht atrophisch sind.

Dessauer (Ingenieur, Aschaffenburg): In einer weichen Röhre entstehen viel mehr Strahlen als in der harten und daraus erklärt sich der verschiedene Grad der Wirksamkeit. 
Demetriades (Jassy): Mitteilung über einen Fall von Lues hereditaria tarda und Laryngopathia luetica.

Patient bis zum 10. Lebensjahre gesund, erkrankte unter Schmerzeu im linken $F u n$ und in der linken Hand. Ein Jahr später traten Geschwüre auf der Stirn, Händen und FüBen auf, die unter Narbenbildung abheilten. Um diese Zeit begannen die Beine sich zu krümmen. Im Jahre 1901 wurde Patient heiser und es traten schwere asphyktische Zustände auf. Die Tibien des Kranken sind nach der beschriebenen Lues hereditaria tarda Fournier gestaltet. Unter eingeleiteter antispezifischer Kur besserten sich die Erscheinungen.

MLerk (Graz): Kritische Betrachtungen über die Symptomatologie der Herpeserkrankungen und die Beziehungen von Hautkrankheiten zu hautfernen Lymphangioititiden.

An der Hand eines von Horstman geschilderten Falles bespricht M. die Symptome des Herpes vom Beginne an. Zuerst ist eine schwache diffuse Rötung, nach mehreren Tagen erst treten stärker rote Stellen auf, am 14. Tage erscheinen die Bläschen. Es ist also eine langandauernde Saftflut dem Bläschenausbruche vorangegangen. Anfangs ist Hyperästhesie, später Anästhesie.

Vortr. wendet sich dann dem Bilde der kutanen Lymphgefäßentzündung zu und sucht die Fragen zu behandeln, wie etwa bei hautfernen Lymphgefäßentzündungen der Verlauf sein dürfte, durch welche Ursacheu sie hervorgerufen werden können und von welchen Symptomen sie begleitet seiu mögen.

Zosteren, welche von motorischen Lähmungen begleitet sind, sind in der Literatur bekannt. Man wird die Lähmung im Gebiete desjenigen motorischen Nerven erwarten müssen, der mit dem ergriffenen sensorischen Nerven parallel läuft. Am einfachsten wird die Erklärung bei gemischten Nerven. Ein Herpes facialis vorgesellschaftet mit einer Facialislähmung ist als einheitlicher pathologischer Prozeb ganz gut denkbar, wenn man sich vorstellt, da6 z. B. eiue Erkältung eiven Katarrh der Nasenschleimhaut, diese eine Lymphangioitis und eine Mitaffektion des Trigeminus und des Facialis herbeiführt. Und weon man sieht, daB auf der einen Seite eine Neuritis eines sensiblen Nerven von einem Herpes gefolgt ist, daß eine Neuritis eines gemischten Nerven Herpes und Läbmung erzeugt, dann werden die so häufig vorkommenden Lähmungen rein motoriseher Nerven gleichsam als das reine Bild derselben Erkrankung in der motorischen Sphäre erscheinen, wie sonst eine sensorische Neuritis das reine Bild eines Herpes bedingt. Dem Herpes sensorius stünde dann ein Herpes "motorius" gegenüber. Je zentraler die Neuritis, desto mehr Hautgebiete werden erkranken, desto deutlicher das Bild des Zoster. Je peripherer, des to mehr entstebt der Herpes vulgaris. Ursachen für Herpes und Zoster sind dieselben wie für Neuritis und die Lymphangioititideu spielen als Vermittler eine vielfach verkanute Rolle.

Geyer ( $Z$ wickau): Demonstration einer eigenartigen Lichenerkrankung-

G. stellt einen Patienten vor, der nehen den Erscheinungen von Arsenmelanose und Arsenkeratose eine Affektion zeigt, welche knötchenförmige, starl schuppende Einzeleffloreszenzen auf leicht geröteter oder normaler Haut bildet, die auf Arsenbehandlung zwar schwinden, aber nach Aussetzen der Medikation immer wieder auftauchen. Er glaubt, daß diese Erkrankung der Gruppe des Lichen ruber acuminatus oder der Pityriasis rubra pilaris oder der Keratosis follicularis rubra zuzurechnen ist.

Diskussion: Pick (Prag) hält den Fall für eine Psoriasis mit einem seltener vorkommenden Ausgang der Hyperkeratose. Er empfiehlt lokale Behandlung mit Chrysarobin. 
Ehrmann (Wien) ist der gleichen Ansicht.

Ullmann (Wien): Weitere Mitteilungen über Wirkungen konstanter Wärme höherer Temperaturen auf die Haut; Demonstration eines Hydrothermoregulators und Nebenapparates für die lokale Thermotherapie.

Die lokale rationelle Thermotherapie besteht in der Anwendung von temperierbaren Kataplasmen, deneu man durch beliebig lange Zeit durch das Prinzip der Autoregulierang dieselbe Temperatur mitteilen und in derselben Höhe beibehalten lassen kaun. Das U.'sche Verfahren bietet die Gewähr, das meiste und überhaupt das wünschenswerte an Temperatur durch beliebige Zeit auf die betreffende Körperstelle einwirken lassen zu können, ohne die Gefahr der Schädigung des Protoplasma und deren Umgebung. Weiterhin gewährt es die Möglichkeit der Applikation von Wärme auf alle Körperregionen, auf die Haut und alle Höhlungen, Nase, Ohr, Vagina, Rektum u. a. Als Wärmezuleitungskörper dienen Röhren, Schläuche, Ballons aus Gummi oder Metall. U. bespricht dann des Genaueren die von ihm konstruierten Apparate und die Indikationen für die Anwendung dieser Behandlungsweise.

An der sich anschließenden Diskussion beteiligten sich Freund. und Ullmann.

\section{Rosenstadt (Karisbad): Öber den Verhornungsprozeß.}

R. stellte seine Untersuchungen an dem Eizahn, einem embryonalen Gebilde des Hühnchens an und kam dabei zu folgendem Resultate: Die nach außen gerichteten Partien des Eizahnes bestehen aus vollständig homogenen Zellen, die stellenweise miteinander verschmelzen. Setzt man Schnitte von solchen Stellen der Wirkung einer kräftigen Verdauungsflüssigkeit aus, so kommt es zu einer ganz geringfügigen Aufhellung. Die homogenen Zellen mit ihren stellenweise durchschimmernden Kernen bleiben aber vollkommen unverdaut. Es ergibt sich somit, daß hier das ganze Protoplasma zusammen mit dem Kerne der Verhornung unterliegt. Dieser Befund ist insoferne von Interesse, als er zeigt, daß es noch einen anderen Modus der Verhornung gibt, bei dem nicht allein der Mantel der Zelle verhornt, sondern die ganze Zelle diesem Prozeß anheimfällt und zwar unter vorheriger Ausscheidung von spezifischen Körnern, die vielleicht als die Ursache des ganzen Prozesses anzusehen sind.

Diskussion: Merk (Graz) erinnert, daß er nebeu Rabl an ausgebildeten menschlichen Hornzellen Plasma- und Epithelfasern nachgewiesen hat und daß die Ansicht, als fehlten diese Fasern in Hornzellen, unrichtig sei.

Waelsch (Prag): Über chronische Prostatitis.

W. konnte bei 200 chronischen Gonorrhoen in $81^{\circ}$ o chronische Prostatitis konstatieren. Von diesen 200 Fällen waren $86 \%$ Urethritis posterior ; es ist also fast jede chronische Posterior mit Prostatitis kompliziert. Die Diagnose Prostatitis darf nur gestellt werden auf Grund des mikroskopischen Befundes im Sekrete der Drüse; die rektale Untersuchung allein reicht nicht aus. Der gonorrhoische Prozeß in der Urethra kann ganz ausgeheilt sein und doch kann die Prostatitis bestehen, die zur Reinfektion oder Infektion der Frau Veranlassung geben kann. Die chronische Prostatitis ist in vielen Fällen die Ursache der Rezidive der chron. Gonorrhoe oder ihrer geringen Beeinflussung durch die Therapie. Die in die Urethra posterior nach Massage der Prostata deponierten medikamentösen Lösungen dringen nicht in die Ausführungsgänge derselben ein; ihre nicht zu leugnende Wirkung dürfte im wesentlichen eine derivatorische sein.

Goldberg (Wildungen): Kleine Mitteilungen über Prostatitis. 
1. Diagnostisches: a) In der überwiegenden Mehrzabl der Fälle kann, nachdem die Harnröhre durch Miktion gereinigt ist, ein Irrtum über die Provenienz des durch Expression gewonnenen Eiters aus der Prustata nicht entstehen, denn der Unterschied zwischen den spärlichen, etwa nach Miktion noch in der Harnröhre verbliebenen Resten und den Massen von Eiterzellen, die bei Expression erscheinen, ist durchaus unverkennbar. b) Bei akuten profusen Eiterungen aus Urethra oder Vesica und negativem Palpationsresultat, ferner bei chronischer Cystitis und Retentio urinae, endlich wenn die Provenienz der Gonokokken aus der Prostata bewiesen werden soll, sind außer der Miktion vorgängige gründliche Reinigungen der Urethra und Blase notwendig, um Verwechslungen mit Harnröhren- oder Blaseneiter zu vermeiden.

2. Prostatitis mit Blutung. Bei akuter Prostatitis sind Blutungen sehr seiten. $G$. sah sie in etwa 5 von 75 Fällen; zweimal wurde aus Hämospermie bei Vorhandensein von Prostatitis und Fehlen ander weitiger Blutungsursachen, dreimal aus dem Blutgehalt des exprimierten Sekretes die hämorrhagische Prostatitis diagnostiziert. Bei chronischer Prostatitis ist die Blutbeimengung zam Sekret nicht so häufig; er fand sie unter 100 Fällen 5mal. Die Blutung hörte immer im Laufe der Be. handlung auf. Die Prostatitiden wurden stets gebessert; die Gonorrhoen waren stets schwere. Von den akuten war eine mit Tripperrileumatismus kompliziert, drei durch Persistenz der Gonokokken im Prostatasekret bis in den 7. Monat gekennzeichnet.

3 Therapeutis ches. Terminale Hämaturien bei subakuter Urethrocystitis können mehrfach nach sehr langern Bustand durch Kombination von I'rostataexpression mit Spülung zur Hsilung kommen. Birnanuell, rektoabdominal, sowie bei erhöhter Stellung kann man am liegenden Patienten anderweitig nicht erreichbare Prostatapartien exprimieren. Die Galvanokaustik nach Bottin $\mathrm{i}$ ist bei chronischer Prostatitis irrationell und zwecklos.

4. Prostatitis und Sterilität. Der behauptete deletäre EinfluB der Prostatitis auf die Potentia generandi durch Abtötung der Spermato. zoen ist bis nun unerwiesen.

Diskussion: De 1 ban co (Hamburg): Ebenso oft wie die Prostata, sind die Samenblasen bei der chroniscben Gonorrhoe beteiligt. Fr lält die chronische Prostatitis oft genug für bedeutunglos. Die lokale Behandlung der Prostata züchtet Neurastheniker.

Galewsky (Dresden) schließt sich Waelsch an. Die Prognose der Urethritis anterior ist günstig, die der posterior ungünstig.

Pincus (Berlin): Selbst nach den gründlichsten spülungen gelingt es zuweilen nicht, sicher zu entscheiden, ob der im Sekrete vorhandene Eiter aus der Prostata stammt oder nur aus der Urethra anterior.

Ehrmann (Wien) weist darauf hin, daB beim Ausdrücken der Prostata immer noch Leukozyten bleiben, die zwischen den Zellen liegend, der Expression entgehen.

Ullmann (Wien) glaubt, daß Waelsch um einige Prozente zu hoch gegriffen hat; er möchte jene Fälle, die palpatorisch keinen Befund geben, nicht hinzugezählt wissen. Therapeutisch empfiehlt or die Anwendung protrahierter konstanter Wärme.

Lö w en heim (Liegnitz) beobachtete mehrere Fälle von Prostatitis ohne bestehende Posterior und erklärte sich dies damit, daß die Entzündung der Prostata sprungweise, vielleicht auf dem Lymphwege entstanden sei. 
180 Verhandl. der 74. Versammlung deutscher Naturf. u. Ärzte.

Wa else h (Schlußwort): In zirka $2 \%$ der chron. Prostatitiden lassen. sich Gonokokken nachweisen. Das Fehlen der Gonokokken möchte er nicht als absoluten Beweis für die Nichtinfektiosität des Prostatasekretes ansehen, aus denselben Gründen, die bei der Beurteilung von eiterzellenhaltigen Fäden der Urethra maßgebend sind. Daß, wie $L$ ö w en h e i m sagt, Epididymitis und Prostatis ohne Urethrititis posterior auftreten kann, ist ihm bekannt. Anders liegen aber die Verhältnisse bei chronischer Prostatitis, die ja zumeist sich nicht aus einer akuten entwickelt, sondern ganz langsam und chronisch im Anschlusse an chronische Urethritis posterior entsteht.

Hierauf hält der zweite Karlsbader Einfübrende Herr Dr. Hugo Guth eine von lebhaftem Beifall gefolgte Ansprache an die Sektionsteilnehmer, in welcher er, nach einem kurzen Rückblicke auf die Verhandlungen der Sektion, zunächst dem Vorstande der Prager Klinik Herrn Prof. Pick und dessen Assistenzärzten, sodann allen Vortragenden und Teilnehmern an den Arbeiten der Sektion den Dank ausspricht und die Verhandlungen der Sektion für Dermatologie und Syphilis an der 74. Versammlung der Deutschen Naturforscher und Ärzte als g e s c h l o s se n erklärt. 